\title{
Genome sequencing of 39 Akkermansia muciniphila isolates reveals its population structure, genomic and functional diverisity, and global distribution in mammalian gut microbiotas
}

\author{
Xianfeng Guo ${ }^{1+}$, Shenghui $\mathrm{Li}^{2+}$, Jiachun Zhang ${ }^{1}$, Feifan $\mathrm{Wu}^{1}$, Xiangchun $\mathrm{Li}^{2}$, Dan $\mathrm{Wu}^{1}$, Min Zhang ${ }^{1}$, Zihao Ou', \\ Zhuye $\mathrm{Jie}^{2}$, Qiulong $\mathrm{Yan}^{3}$, Peng $\mathrm{Li}^{2}$, Jiangfeng $\mathrm{Yi}^{4}$ and Yongzheng Peng ${ }^{1^{*}}$ (D)
}

\begin{abstract}
Background: Akkermansia muciniphila is one of the most dominant bacteria that resides on the mucus layer of intestinal tract and plays key role in human health, however, little is known about its genomic content.

Results: Herein, we for the first time characterized the genomic architecture of A. muciniphila based on whole-genome sequencing, assembling, and annotating of 39 isolates derived from human and mouse feces. We revealed a flexible open pangenome of A. muciniphila currently consisting of 5644 unique proteins. Phylogenetic analysis identified three species-level A. muciniphila phylogroups exhibiting distinct metabolic and functional features. Based on the comprehensive genome catalogue, we reconstructed 106 newly A. muciniphila metagenome assembled genomes (MAGs) from available metagenomic datasets of human, mouse and pig gut microbiomes, revealing a transcontinental distribution of A. muciniphila phylogroups across mammalian gut microbiotas. Accurate quantitative analysis of $A$. muciniphila phylogroups in human subjects further demonstrated its strong correlation with body mass index and anti-diabetic drug usage. Furthermore, we found that, during their mammalian gut evolution history, A. muciniphila acquired extra genes, especially antibiotic resistance genes, from symbiotic microbes via recent lateral gene transfer.
\end{abstract}

Conclusions: The genome repertoire of A. muciniphila provided insights into population structure, evolutionary and functional specificity of this significant bacterium.

Keywords: Akkermansia muciniphila, Genome diversity, Population structure, Mammalian gut microbiota, Antibiotic resistance

\section{Background}

Akkermansia muciniphila is a commensal anaerobe that is found to reside in the intestinal tract of more than $80 \%$ of human population [1-3], constituting $1-4 \%$ of the total bacterial cells in the healthy adult feces. The presence of $A$. muciniphila is also detected in feces from

\footnotetext{
* Correspondence: yzpeng1981@126.com

${ }^{\dagger}$ Equal contributors

'Department of Laboratory Medicine, Zhujiang Hospital, Southern Medical University, Guangzhou 510282, China

Full list of author information is available at the end of the article
}

newborns and the incidence increases rapidly during the first year of life [4], reaching $0.9 \%$ and $1.6 \%$ of relative abundance in 6- and 12-month-old infants, respectively [5]. Specifically, Akkermansia-like organisms are widely distributed in the intestines of non-human mammals such as lemur, gorilla [6] and mice [7], as well as other vertebrates such as chickens [8] and zebrafish [9].

When residing on the mucus layer of intestinal tract [10], A. muciniphila uses intestinal mucins, the highly glycosylated proteins of the epithelial mucus layer, as its major carbon and nitrogen sources that leads to the 
production of acetate and propionate, which are the important energy sources of human intestine epithelial cells [10]. This unique mucin-degrading feature makes A. muciniphila a modulator for gut homeostasis via strengthening the integrity of the epithelial cell layer [11] and regulating the gut barrier function $[12,13]$. Recent studies demonstrated that A. muciniphila is beneficial to host health; e.g., A. muciniphila treatment reversed high-fat-diet-induced obesity and metabolic disease in mice [14, 15], and an increase in the Akkermansia spp. population induced by metformin treatment improved glucose homeostasis in mice [16]. Moreover, growing evidences showed that the abundance of $A$. muciniphila was inversely correlated with body weight $[17,18]$, type 1 diabetes [19], inflammatory bowel disease [20], and autism [21], in both mice and humans. Other studies found that $A$. muciniphila was enriched in type 2 diabetes [22] and colorectal cancer subjects [23].

The first and only available $A$. muciniphila genome, ATCC BAA-835, was sequenced in 2011 [24], comprising one circular chromosome of $2.66 \mathrm{Mbp}$. This genome showed distinct phylogenetic features in contrast with other genomes of the Verrucomicrobia phylum, as only $29 \%$ of genes were shared with its closest relative, varying largely in $\mathrm{G}+\mathrm{C}$ content and genome size [24]. Specifically, when comparing all available bacterial or archaeal genomes in the national center for biotechnology information (NCBI) database, no non-Akkermansia sequences hit more than $90 \%$ of nucleotide identity with the whole $A$. muciniphila genome, indicating a unique and conservative evolutionary status of this bacterium [24] (we confirmed this result in July 2017 with 23,802 sequenced genomes). These evidences suggest that $A$. muciniphila has independently evolved for a long period, during which at least five divergent clades emerged, as revealed by phylogenetic analysis based on Akkermansia 16S rRNA gene sequences from mammalian-derived samples [8]. Furthermore, in our previous study [25], we isolated 22 A. muciniphila strains from human fecal samples and revealed 12 distinct subtypes via DNA fingerprint analysis. This motivated us to build the genome repertoire of $A$. muciniphila and investigate its population structure and characteristics on genomic level.

Here, we whole-genome shotgun sequenced and analyzed 39 new A. muciniphila strains isolated from human $(n=33)$ and mouse $(n=6)$ fecal samples. We also reconstructed 106 newly $A$. muciniphila draft genomes from extensive available metagenomic datasets of human (including Chinese, European and American), mouse and pig gut microbiomes. Our results showed that the genome contents of A. muciniphila are flexible with an open pangenome and frequently acquire genes from other bacteria via recent lateral gene transfer (LGT). We revealed a remarkably high genetic diversity within A. muciniphila and accordingly classified this species into three species-level phylogroups. Based on this enormous amount of genomic data, we accurately quantified the occurrence rate and abundance of $A$. muciniphila phylogroups in mammalian gut microbiomes, and investigated its association with body mass index and anti-diabetic drug usage in human subjects. Our results thus provided the genomic and evolutionary landscape of A. muciniphila.

\section{Results}

\section{Overview of the A. muciniphila genomes}

A total of 39 A. muciniphila isolates were collected and whole-genome shotgun sequenced by Illumina approach. De novo assembly of their genomic data revealed varying genome size ranging from 2.65 to $3.20 \mathrm{Mbp}$ (averaging $2.86 \mathrm{Mbp}$, Additional file 1: Table S1). The genome sizes of almost all $(38 / 39)$ isolates were larger than that of ATCC BAA-835, indicating that extensive genomic contents of $A$. muciniphila were unexplored previously.

The number of protein-coding genes of the 40 available A. muciniphila genomes (39 newly isolates and strain ATCC BAA-835) varied from 2138 to 2664 (averaging 2370, Additional file 1: Table S1). To assess the gene contents of A. muciniphila, we identified a pangenome containing 5644 unique protein-coding genes among these genomes. The gene accumulation curve fit the Heap's low $(n=\kappa N \gamma)$ with parameters $\gamma=0.25 \pm 0.02$ (95\% confidence interval) (Fig. 1a), and the occurrence of new genes fit the power low $\left(n \sim N^{-} \alpha\right)$ with exponent $\alpha=0.70 \pm 0.04$ (Additional file 2: Figure S1). Both findings indicate an open pangenome of $A$. muciniphila. 34 unique genes will potentially be added along with the availability of additional genomes. The gene occurrence plot (Fig. 1b) showed a core-genome containing 1275 genes that were present in all sequenced strains, whereas the other genes were additional accessory genes that were mostly present in a few of genomes ( $60 \%$ of genes found in $\leq 5$ genomes and one fourth found in only one genome).

\section{Population structure within A. muciniphila species}

To investigate the population structure of $A$. muciniphila, we identified 270,928 high-quality single nucleotide polymorphisms (SNPs) within the core-genome $(1.47 \mathrm{Mb}$ of sequences in total). Such a huge number of SNPs indicated the highest level of nucleotide diversity (184 SNPs $\mathrm{kb}^{-1}$ ) in A. muciniphila compared with other prevalent human gut microbial species [26], which may be facilitated by ancient evolutionary history and large population symbiotic in human and other mammals (see Discussion). Maximum likelihood (ML) phylogenetic analysis of these SNPs (Fig. 2a) identified three major phylogroups (defined as AmI, AmII and AmIII), with 100\% bootstrap support. This separation was also consistent with average 

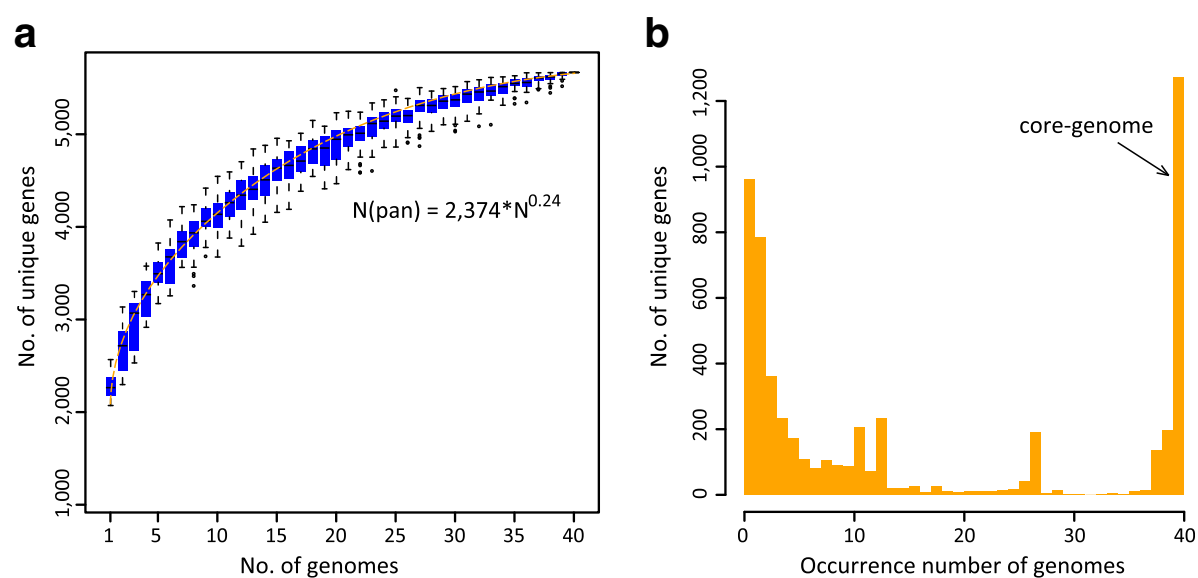

Fig. 1 Pangenome, core-genome and gene occurrence of the 40 analyzed A. muciniphila isolates. (a) Pangenome accumulation curves. The blue boxes denote the number of unique genes discovered with the sequential addition of new genomes. The equations below the graph show parameters for fits to Heap's law, and the positive exponent indicates an open state of the pangenome. (b) Gene occurrence plot shows the core-genome and additional accessory genes of A. muciniphila

nucleotide identity (ANI) clustering and principal components analysis (PCA) based on the whole genomic data (Fig. 2b and Additional file 2: Figure S2). Within each phylogroup, the ANI between genomes was $97.2-100 \%$, whereas ANI between phylogroups was $86.8-91.5 \%$. Based on the between-phylogroup nucleotide conservation of $96 \%$, which is normally used as a threshold for prokaryotic species definition [27], we noted that AmI, AmII and
AmIII are distinct species and these phylogroups constitute discrete bacterial populations that are evolving independently. Conversely, these phylogroups also shared highly consistent phenotypic characteristics [25], habitat, and conservative 16S rRNA genes (nucleotide similarity $>99 \%$ between any two genomes).

To examine the functional significance, we annotated the A. muciniphila pangenome using the eggNOG
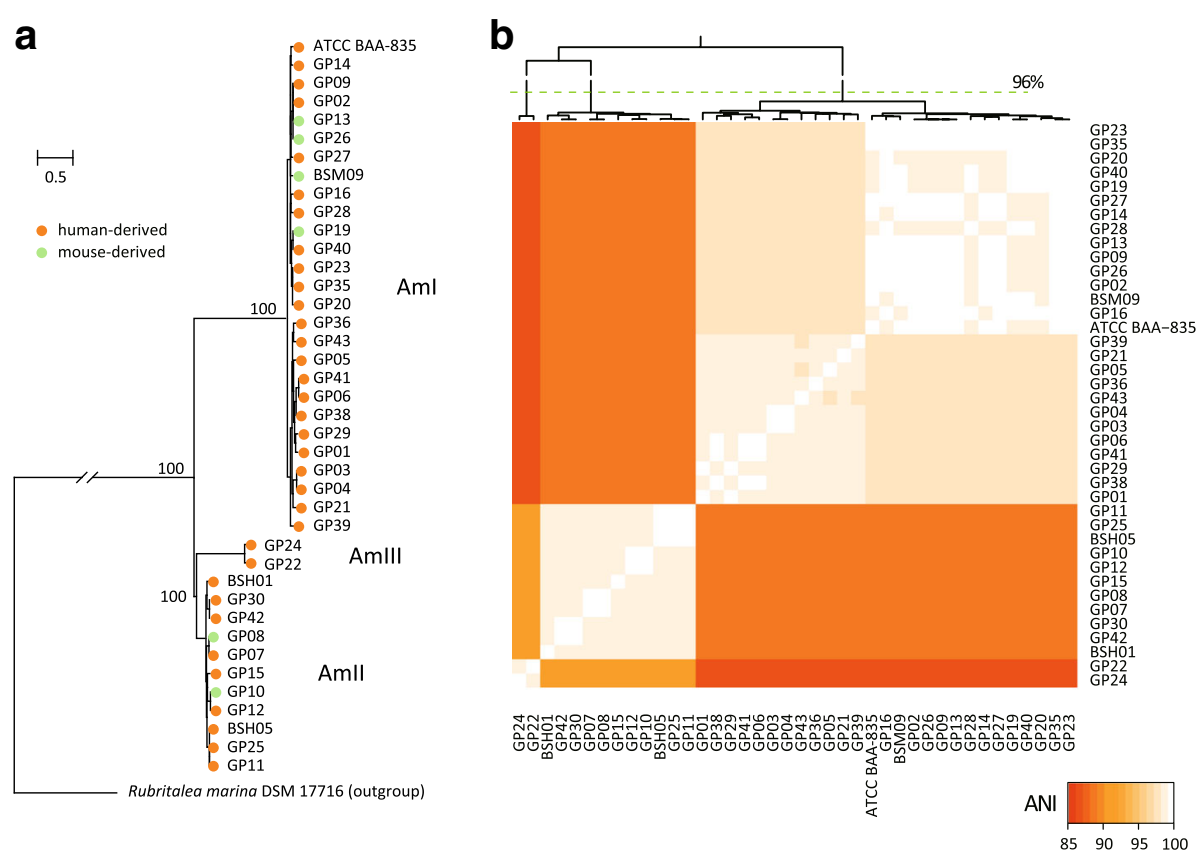

Fig. 2 Phylogroups of A. muciniphila. (a) Maximum likelihood phylogenetic tree based on core genome SNPs of 40 A. muciniphila isolates. The tree is inferred using RAxML with 1000 replications, with Rubritalea marina DSM 17716 as outgroup species. Bootstrap support values are shown for the separation nodes of the main phylogroups. (b) Hierarchical clustering of isolates based on whole-genome level average nucleotide identity (ANI). Pairwise ANI between isolates is shown as a heat map 
(evolutionary genealogy of genes: Non-supervised Orthologous Groups) [28] and KEGG (Kyoto Encyclopedia of Genes and Genomes) [29] databases. Due to the lack of extensive functional research on Akkermansia species or its evolutional related taxa, only $29.6 \%(1668 / 5644)$ genes could be assigned into provisional function, and the others were mostly "hypothetical proteins". All A. muciniphila strains carried a large proportion of proteins that are involved in genetic information processing (translation, DNA replication and repair), membrane transport, signal transduction and metabolism of a variety of compounds (Additional file 2: Figure S3). However, each phylogroup carried a unique subset of proteins when comparing their functional profiles. Based on the PCA analysis of KEGG pathways (Fig. 3a), a dramatic divergence was found between the genomes of phylogroup AmI and AmII/AmIII, whereas AmII and AmIII were relatively closer, possibly due to their evolutionary relationship. The AmI genomes were enriched in "phosphatidylinositol signaling system", "degradation of aromatic compounds", "two-component system", "ABC transporters", etc.; while the AmII/AmIII genomes were enriched in "amino sugar and nucleotide sugar metabolism", "glycosphingolipid biosynthesis - globo series", "sulfur relay system", "fructose and mannose metabolism", etc. (Fig. 3b). A. muciniphila is commonly known as a mucin-degrading bacteria in the human fecal microbiota, likely depending on its possession of abundant extracellular glycosidases [24]. When we annotated the carbohydrate-active enzymes (CAZymes) on the A. muciniphila genomes, AmI also showed substantial difference from that of AmII and AmIII (Additional file 2: Figure S4). Generally, the AmII/AmIII genomes carried larger number of CAZymes than AmI did (average number of
CAZymes, 210 vs. $191 ; P<0.001)$, especially the glycosyl transferase family 4 (GT4) (average number of genes, 35 vs. $22 ; P<0.001$ ) which is involved in the biosynthesis of several oligosaccharides. This result indicated that AmII and AmIII are more versatile in metabolizing carbohydrates and substrates, which could also be reflected by the enrichment of polysaccharide (e.g. fructose and mannose) metabolism in KEGG pathways.

\section{Global distribution of $A$. muciniphila phylogroups in mammalian hosts}

To explore the genomic content of $A$. muciniphila in human and other mammalian gut microbiotas, firstly, public metagenomic datasets used in the cataloguing of gut microbiomes in human (1267 fecal samples, from Chinese, $n=368$ [22]; European, $n=760$ [30]; and American, $n=139$ [31]), mouse (184 samples [32]) and pig (290 samples [33]) were queried. Using a simplified approach based on the genomic unique of $A$. muciniphila (see Methods), we reconstructed 106 metagenome assembled genomes (MAGs) from these microbiomes (99 in human, 3 in mouse, and 4 in pig), and obtained an average of $2.82 \mathrm{Mbp}$ (average contigs number of 235 , average N50 length of $42.9 \mathrm{kbp}$ ) genome size for each MAG (Additional file 3: Table S2). Phylogenetic analysis of the MAGs was performed combined with 40 known A. muciniphila isolates. Based on the ML-tree (Fig. 4) and a nucleotide conservation threshold of $96 \%$, the majority (103/106) of newly constructed genomes were assigned into the major A. muciniphila phylogroups, AmI and AmII. Of the other 3 unplaced MAGs, two (from European) were highly homologous to AmI and one (from American) was highly homologous to AmIII.
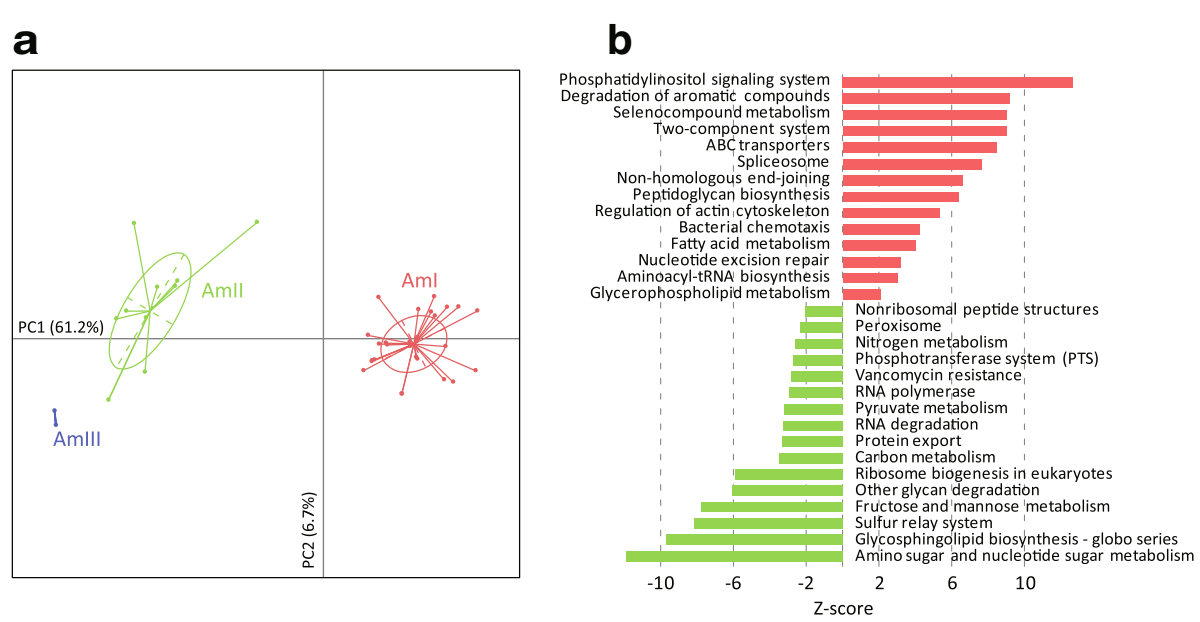

Fig. 3 Functional difference between A. muciniphila phylogroups. (a) Principal components analysis based on the KEGG profile. Isolates on the first and second principal components (PC1 and PC2) are plotted by nodes. Lines connect isolates in the same groups, and colored circles cover the isolates near the center of gravity for each group. (b) The significantly different pathways between Aml and Amll/III. Pathways with false discovery rate (FDR) corrected $P$ value $(q)$ of $<0.05$ (converted to Z-score; two-tailed Fisher's exact test) are shown 


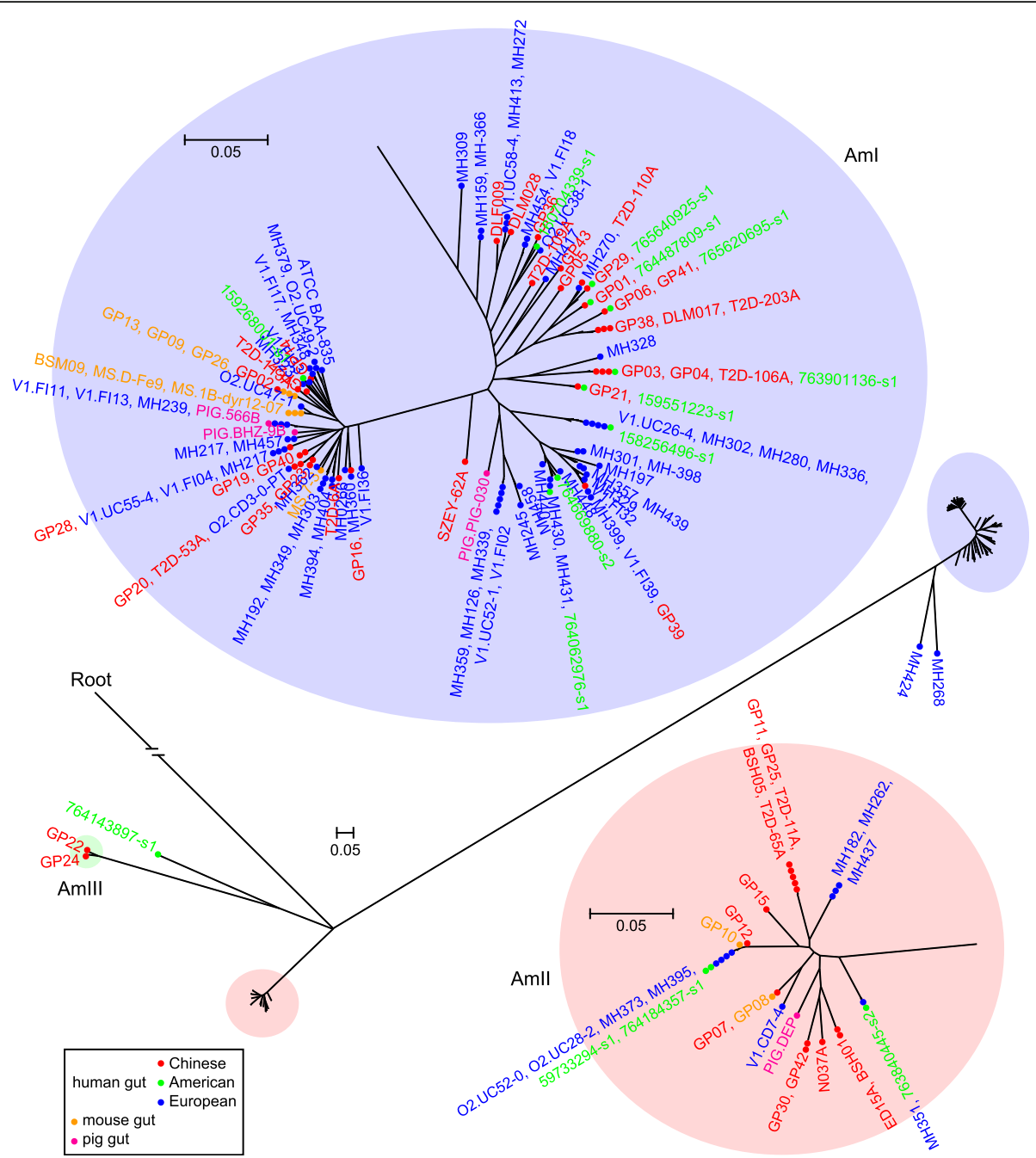

Fig. 4 Phylogroups of A. muciniphila strains derived from human and other mammalian gut microbiotas. Maximum likelihood phylogenetic tree based on core-genome SNPs of 40 A. muciniphila isolates and 106 new constructed genomes (MAGs). The tree is inferred using RAxML with 1000 replications
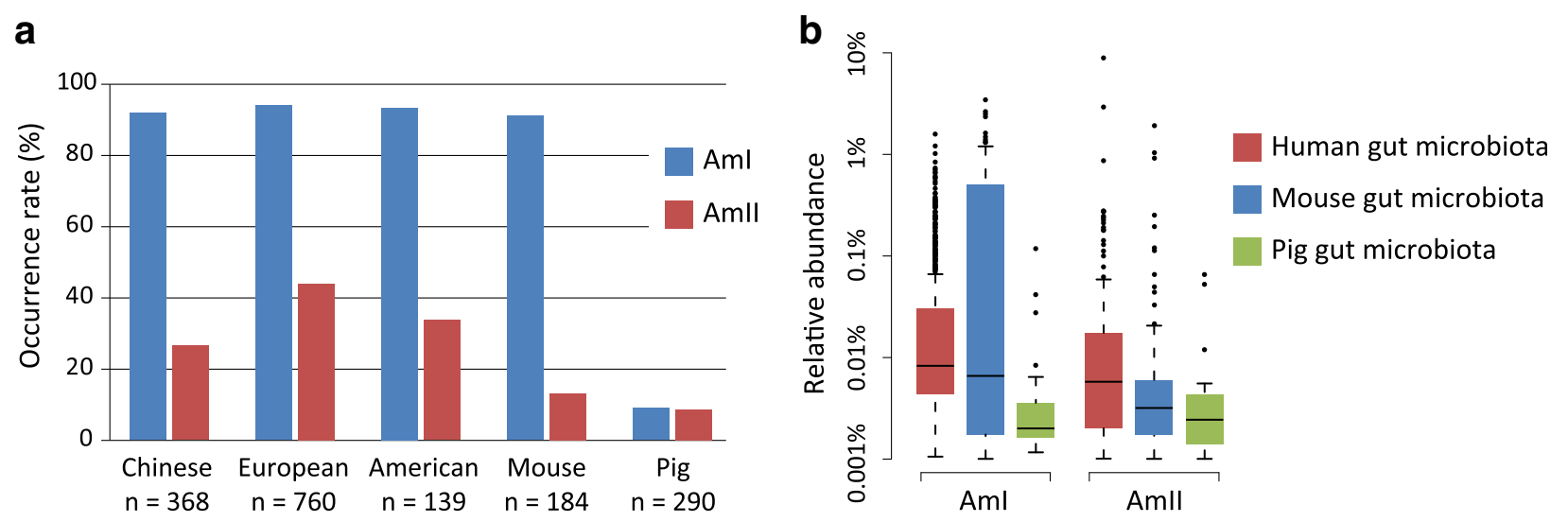

Fig. 5 Distribution of A. muciniphila phylogroups in mammalian gut microbiomes. (a) Occurrence rate in the Chinese, European and American population and other mammalian gut microbiotas. (b) Relative abundance of A. muciniphila phylogroups in selected samples 
Both AmI and AmII contained MAGs from three human populations, mouse and pig (Fig. 4), demonstrating that the distribution of $A$. muciniphila phylogroups were transcontinental and across mammalian hosts.

Such enormous genomic data enabled us to accurately quantify the occurrence rate and abundance of $\mathrm{A}$. muciniphila phylogroups in human and other mammalian gut microbiotas. Of the three phylogroups, we revealed that AmI was the most frequently occurring phylogroup which was found in $93 \%$ of all tested human population (minimum relative abundance $>0.01 \%$ in metagenomic sample, and covering $>10 \%$ of the A. muciniphila coregenome), $91 \%$ of mice and $9 \%$ of pigs (Fig. $5 \mathrm{a}$ ). AmII was also frequently found in human gut, with higher occurrence rate in European (44\%) than in Chinese (27\%; $P<0.001$, Fisher's exact test) and American (33\%; $P=0.02$ ). However, AmII was less frequently found in mouse (12\%; $P<0.001$ compared to human microbiota) and pig $(9 \% ; P<0.001)$ gut microbiotas. Comparing of relative abundance showed AmI had higher abundance in mouse gut than in human and pig, whereas AmII majorly resided in human gut (Fig. 5b).

\section{Abundance of $A$. muciniphila associated with obesity and anti-diabetic drug}

Recent studies explored the relationship between $\mathrm{A}$. muciniphila and obesity and other diseases [14, 18, 34]. In this study, we expanded the analysis of the correlation between relative abundances of A. muciniphila phylogroups (AmIII was not analyzed for its rare occurrence rate) and host phenotypes, including gender, age and body mass index (BMI), in the phenotype-available Chinese individuals. No significant correlation was found between gender, age, BMI and the relative abundance of $A$. muciniphila phylogroups (Additional file 2: Figure S5a-b). However, a significant decrease of A. muciniphila was found in individuals with BMI of over $30(P<0.01$ for both AmI and AmII, MannWhitney $U$ test), consistent with previous study that showed decrease of $A$. muciniphila in severely obese individuals [18]. We also validated the recent study [35] by revealing a significant enrichment of $A$. muciniphila in gut microbiota of the anti-diabetic metformin treated individuals compared with the untreated diabetic or healthy individuals $(P=0.007$, Mann-Whitney $\mathrm{U}$ test after adjusted gender, age and BMI; Additional file 2: Figure S5c). Specially, this phenomenon was only found in AmI.

\section{A. muciniphila's acquisition of antibiotic resistance genes from co-ecological species}

As the only Verrucomicrobia bacteria resident in human and other mammalian guts, A. muciniphila showed drastic differences in morphology and life cycle compared with other Verrucomicrobia species [36, 37], which was assumed to be related with its distinct adaption during mammal-associated evolution. We assumed that the $A$. muciniphila strains gained genes from other species via lateral gene transfer during the evolutionary history mammalian gut. To validate this, we searched for the LGT events by comparing the full gene sets of $40 \mathrm{~A}$. muciniphila strains (39 newly isolates and ATCC BAA-835) with all non-Akkermansia genomes from the NCBI database using BLAST (see Methods). Ten candidate transfer events involving 83 genes (Additional file 4: Table S3) were identified with threshold of more than $85 \%$ similarly with the nearest genomes. All of these transfer events were of low frequency (occurring in less than four strains) and located in the terminal of phylogenetic tree, indicating that LGT occurred recently. A. muciniphila acquired extra genes from a wide range of distinct taxa, including Bacteroides spp., Bifidobacterium longum, and several Firmicutes. Meaningfully, most of these taxa were associated with human gut habitats, suggesting that these genes were gained from the co-ecological species. As a striking example, an LGT event was observed in A. muciniphila GP36 which gained 8 genes from the plasmid pRSF1010 originally found in Salmonella enterica (Fig. 6a). 3 out of 8 genes were antibiotic resistance genes, namely sul2 (sulfonamide-resistant dihydropteroate synthase), aph(6)-Id and $a p h(3 ")-I b$ (aminoglycoside phosphotransferase). Drug sensitive test validated the emergence of resistance against corresponding antibiotics (Fig. 6b). This event indicated that the A. muciniphila species might acquire antibiotic resistance genes via recent LGT to adapt to the high level of antibacterial gastrointestinal environment in modern lifestyle.

\section{Discussion}

In vivo, $A$. muciniphila plays a crucial role in maintaining the integrity of the mucus layer, thereby reducing translocation of proinflammatory lipopolysaccharides and controlling adipose tissue metabolism, decreasing insulin resistance and keeping glucose homeostasis $[14,16]$. Accumulating research evidences uncovered the beneficial effects of A. muciniphila in host [13, 38]. For example, administration treatment of $A$. muciniphila reversed dietinduced metabolic disorders in animal models [14, 15], suggesting its potential function in prevention or treatment of human obesity and other metabolic diseases. Though an increasing number of research reports focus on A. muciniphila ( $>160$ papers by a PubMed research for "Akkermansia muciniphila [title/abstract]" in July 2017), however, only one A. muciniphila strain (ATCC BAA835 ) is isolated and publicly available, due to its arduous colonization conditions in vitro [39]. Here, we analyzed 39 A. muciniphila strains isolated from human and mouse feces via our recently developed methodology [25], as well as 106 new MAGs assembled from metagenomic datasets of human, mouse and pig feces samples. 


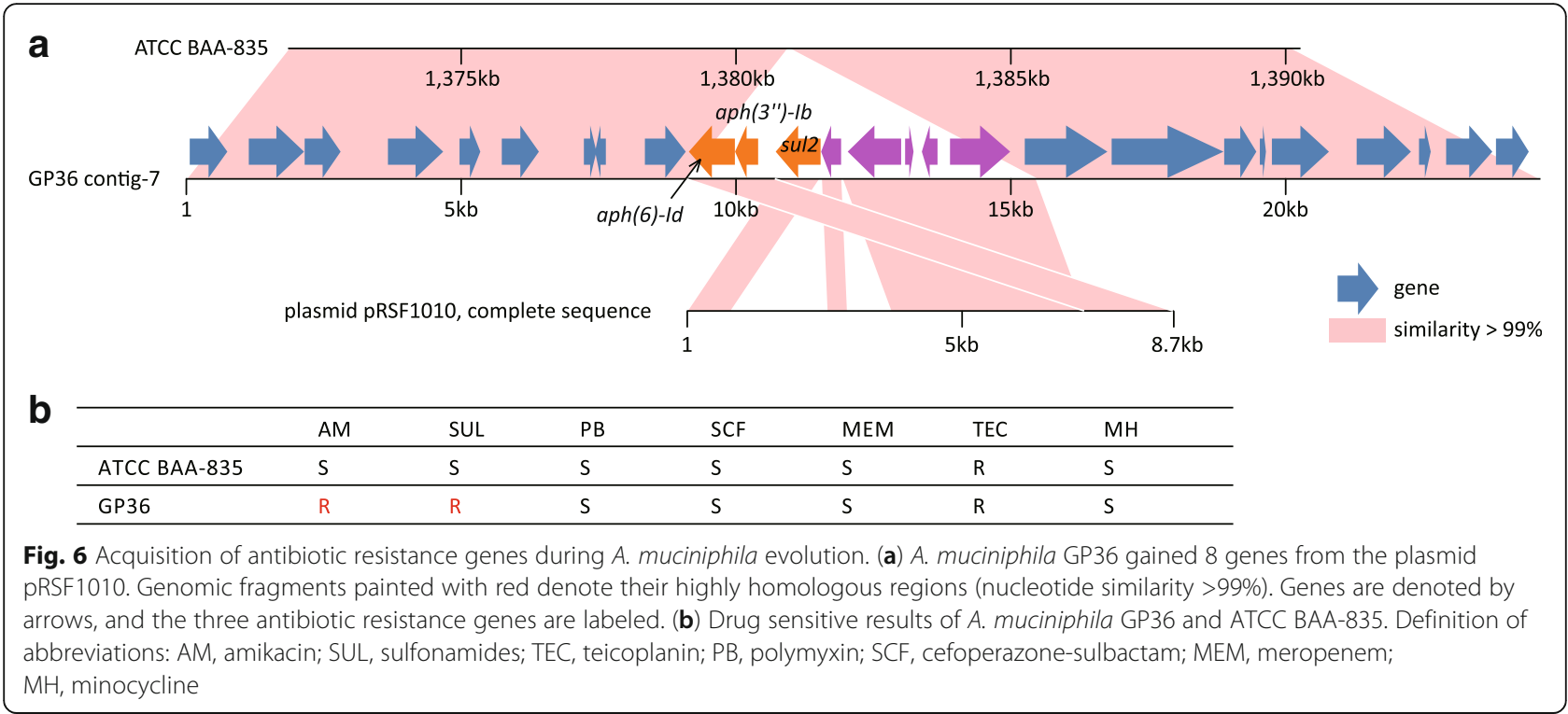

For the first time, we revealed the genomic diversity and population structure of $A$. muciniphila, and identified three phylogroups, AmI, AmII and AmIII, within $A$. muciniphila. 113 of 146 (77\%) known genomes were assigned into AmI, while 28 (19\%) were assigned into AmII, with two AmIII genomes and three unplaced genomes. Phylogroups show high genomewide nucleotide diversity and distinct metabolism and function profiles (Fig. 2 and Fig. 3). Strains within each phylogroup are highly similar in nucleotide sequences (ANI > 97.2\% between any two genomes), whereas strains between phylogroups are divergent (ANI < 91.5\%). When using the nucleotide conservation of $96 \%$ as a threshold for prokaryotic species definition [27], these phylogroups are likely to be defined as different species. However, there are no obvious mechanistic barriers to gene flow between AmI, AmII, and AmIII. Indeed, we observed a number of gene flow events and several recombination events between three phylogroups (Additional file 2: Figure S6), suggesting a possible gene exchange and homologous recombination. Thus, we leave the species definition of $A$. muciniphila for future experimental and/or phenotypic investigation. Moreover, previous study [8] had divided the Akkermansia phylogenetic tree (based on $>400$ full-length 16S rRNA gene sequences) into five clades, while the conceivable $A$. muciniphila lineages were assigned into two distinct clades, clade 1 (16S rRNA gene similarity with strain BAA-835, 97$100 \%$ ) and clade 4 (95-99\%). Although A. muciniphila strains in our study was widely distributed in global human, mouse and pig, the $16 \mathrm{~S}$ rRNA gene sequences of all strains were quite conserved, suggesting that more $A$. muciniphila phylogroups are still undiscovered, especially in non-human animals.
In functional analysis, genes that encode CAZymes are of particular interested, as them are required to metabolize most of dietary polysaccharides [40]. A. muciniphila genomes carried average 198 CAZymes, exceeding the number of CAZymes per genome for most microbes in the human gut microbiota (e.g. members of Firmicutes, but less than Bacteroidetes) [40], highlighting its predominant role in glycan metabolism for the mammalian hosts. In comparison, the AmII/AmIII genomes carried larger number of CAZymes than AmI did, especially the GT4, which is involved in the biosynthesis of oligosaccharides such as sucrose and mannose. Parallelly, the AmII/AmIII genomes harbored more proteins which belong to the KEGG pathways "amino sugar and nucleotide sugar metabolism", "fructose and mannose metabolism" and "carbon metabolism" (Fig. 3b). Combining of these observations suggested more versatile in metabolizing carbohydrates and substrates for AmII and AmIII. Thus, the functional specificity of A. muciniphila phylogroups would further correlate to their phenotypes or lifestyle, however, systematic investigations of key enzymes and functions might be helpful in the future.

A. muciniphila is widely distributed in intestinal tracts of the animal kingdom, and the Akkermansia genus was considered as an indigenous member of the microbiota resident in various animals [8]. Recent studies revealed that a remarkably low number of microbial genes $(\sim 15,000)$ was shared in the gut microbiomes of human (representing 9.9 million non-redundant genes) [30], mouse (2.6 million) [32] and pig (7.7 million) [33]. Strikingly, one third ( 5000) of these genes belonged to A. muciniphila, indicating that A. muciniphila is a major species that exists in multiple mammalian hosts. 
In our datasets, though the A. muciniphila was detected in $91 \%$ of mouse fecal samples (including all the samples collected from different providers from Europe, China and America [32]), only three MAGs all belonging to the major phylogroup AmI, were identified (Fig. 4), and the others were almost identical to one of them. This demonstrates that the genomic diversity of A. muciniphila in mouse gut microbiota is significantly lower than that in human, probably due to the similar genetic background and laboratorial environment of the mice [32].

During the evolution history, A. muciniphila presents a large proportion of losses of orthologous groups compared with its ancestral genome of the Verrucomicrobia phylum [24] (see Additional file 2: Figure S7 for the phylogenetic tree based on the available species of the Verrucomicrobia phylum and the type strains of A. muciniphila), which was speculatively due to its distinct adaption during host-associated evolution. In this study, we found yet another mechanism of A. muciniphila to adapt the mammalian gut habitat. Several $A$. muciniphila strains acquired extra genes from a wide range of microbial taxa, such as Bacteroides spp., Lachnospiraceae spp., and Bifidobacterium longum. Most of the genes are inside a transposable element that can be transferred between multiple species. A striking example is the acquisition of three antibiotic resistance genes (sul2, aph(6)-Id and $a p h(3 ")-I b)$ in strain GP36 (Fig. 6). These genes are part of the plasmid RSF1010, an $8684 \mathrm{bp}$ broad host range plasmid that can replicate in most Gram-negative bacteria and Gram-positive Actinomyces [41, 42], and can simultaneously be found within integrative conjugative elements or chromosomal genomic islands in both Gram-positives and Gram-negatives [43, 44]. We assume that the acquiring of these genes is required to adapt to the high level antibiotic environments in current human gut.

Based on a recent research, A. muciniphila showed an inverse correlation with body weight in both mouse and human models [15, 18]. We expanded these observations in the Chinese cohorts and further found that both of the two major phylogroups of $A$. muciniphila, AmI and AmII, were associated with obesity (Additional file 2: Figure S5b). Previous studies found that anti-diabetic metformin treatment improved glucose homeostasis in association with increased Akkermansia spp. population in the gut microbiota of mice [16]. Likewise, metformin is also associated with higher relative abundance of $\mathrm{A}$. muciniphila in the human gut [35]. In this study, we found only AmI was associated with metformin treatment in the Chinese diabetic individuals (Additional file 2: Figure S5c), suggesting a different anti-diabetic effect of AmI and AmII. In spite of these findings, more experimental evidences are required.

\section{Conclusions}

This study characterized the genomic architecture of $A$. muciniphila based on whole-genome sequencing of 39 isolates and metagenomic-reconstructing of $106 \mathrm{draft}$ genomes from mammalian gut feces. The genome contents of A. muciniphila are flexible with an open pangenome and frequently acquire genes from co-ecological bacteria via lateral gene transfer. We revealed high genetic diversity of $A$. muciniphila and classified them into three species-level phylogroups. We also quantified the occurrence rate and abundance of A. muciniphila phylogroups in mammalian gut microbiomes, and investigated its association with host phenotypes. In summary, our results demonstrated the notable population genomic diversity, functional specificity, geographically distribution and ecological adaptation of A. muciniphila. The comprehensive genomic framework of A. muciniphila provide solid foundation and practical support for future studies.

\section{Methods \\ Bacterial isolates}

Six strains were isolated from mouse fecal samples while other 34 strains (including A. muciniphila sp. GP37) were isolated from human feces. All 40 strains were isolated as previous described with some modifications [10]. Briefly, about $1.0 \mathrm{~g}$ of fresh feces were collected from mouse (C57BL/6) or outpatients in Zhujiang Hospital and mixed in $5.0 \mathrm{ml} 0.9 \%$ salt solution thoroughly. The suspension was then collected for serial dilutions (10-fold) in $0.9 \%$ salt solution. $1.0 \mathrm{ml}$ of the highest diluted suspension $\left(10^{-6}\right.$ dilution) was inoculated in $9.0 \mathrm{ml}$ mucin medium and then the mixture was kept in anaerobic condition at $37{ }^{\circ} \mathrm{C}$ for about one week until obvious turbidity was observed. The mucin medium was made according to the method previously described [10]. The positive tubes after enrichment were further purified by repeated inoculation on the same medium containing $0.75 \%$ agar (OXOID, England). Single colonies were collected and identified by $16 \mathrm{~S}$ rRNA gene sequencing.

\section{DNA preparation and sequencing}

Bacteria were grown in mucin medium for $48 \mathrm{~h}$ and harvested by centrifuge at $400 \mathrm{~g}$ for $10 \mathrm{~min}$. Bacterial DNA was extracted by Ampure Microbial DNA Kit (Magen, Guangzhou, China) according to maufacturer's instruction. DNA library was constructed using the TruSeq DNA protocol LT Sample Perp Kit following the manufacturer's instruction, and whole-genome shotgun sequenced using the Illumina HiSeq 2500 instrument, which generated a series of 150 bp paired-end reads with expected 250-300 bp insert size for every strain. High quality reads were extracted from the raw Illumina data by trimming the low quality $(\mathrm{Q}<30)$ bases on the end 
of reads and filtering ' $\mathrm{N}$ '-containing, adapter contamination or short length $(<100 \mathrm{bp})$ reads, using the FASTX-Toolkit [45].

Genome assembly, annotation and pangenome analysis. Short reads for each $A$. muciniphila isolate were assembled using Velvet [46], an algorithm for de novo short read assembly using de Bruijn graphs. For each isolate, the procedure was run multiple times using different kmer parameter ranging from 35 to 145 to generate the best assembly result. Then, the raw assembled genome was performed by contig extension and scaffolding by SSPACE [47], and the results were performed by gap closing procedure using GapFiller [48]. The shortest scaffolds were filtered with minimum length threshold of $200 \mathrm{bp}$. The previous finished genome of strain ATCC BAA-835 was downloaded from NCBI bacterial genome database (accession no. NC_010655).

Genomic annotation was implemented using the Prokka [49] pipeline. Prokka used a suite of prediction tools to identify the coordinates of genomic features within contigs, including small rRNA (5S, $16 \mathrm{~S}$ and $23 \mathrm{~S}$ rRNA) using RNAmmer [50], tRNA using Aragorn [51], and Clustered Regularly Interspaced Short Palindromic Repeats (CRISPR) using MINCED [52]. Prokka annotated the protein coding genes in a hierarchical manner: 1), Prodigal [53] identifies the coordinates of candidate genes; 2), the homologous proteins of $A$. muciniphila ATGG BAA-835 was used as trusted annotate from; and 3), the other protein families were searched from bacterial proteins in UniProt and RefSeq databases. After running Prokka, genes were further annotated to eggNOG (evolutionary genealogy of genes: Non-supervised Orthologous Groups, v4.5) [28] and KEGG (Kyoto Encyclopedia of Genes and Genomes, downloaded Feb. 2016) [29] databases using BLASTP (identity threshold of $35 \%$, covering $>70 \%$ of the gene length; -qcov_hsp_perc parameter, 0.7). Remaining genes with no matches in Prokka, eggNOG or KEGG were labeled as "hypothetical protein". Glycoside hydrolase genes were annotated using the Carbohydrate-Active enZYmes (CAZy) [54] database.

Pangenome analysis was performed using Roary [55] with default parameters.

\section{Average nucleotide identity}

Average nucleotide identity (ANI) between two genomes was calculated using the "ANIb" algorithm which uses BLAST as the underlying alignment method $[27,56]$.

\section{SNP calling and phylogenetic analysis}

To detect SNPs in the core genome, paired-end reads of each isolate were mapped using BWA [57] ( $-\mathrm{n}$ parameter, 22; minimal identity of $85 \%$ ) against the reference genome of strain ATCC BAA-835. Mapped were considered only if they matched the core gene regions (1298 genes in
1.95 $\mathrm{Mb}$ of sequences) of the reference. Candidate variants were extracted using SAMtools mpileup [58], followed by BCFtools from the SAMtools package with filtering parameters of variant quality score greater than 50 and mapping quality greater than 30 . Uncertain variant was further filtered if its major allele frequency (as calculated by BCFtools) is less than $80 \%$ or reads supporting number less than 5. Phylogenetic reconstruction was carried out using the maximum-likelihood program RAxML v8.2.4 [59] with a GTR model of evolution, and visualized using MEGA [60]. Robustness of the phylogenetic tree was estimated by bootstrap analysis in 1000 replicates.

\section{Metagenomic data source, draft genome reconstruction and further analysis}

To analyze the distribution of $A$. muciniphila in human and other mammalian gut microbiomes three public metagenomic datasets were included: (i) 1267 human fecal samples provided by $\mathrm{Li}$, et al. [30], which included 368 Chinese subjects (first published in [22], NCBI accession no. SRA045646 and SRA050230), 760 European subjects, and 139 American subjects (first published as part of the Human Microbiome Project [31], data available at http://www.hmpdacc.org/HMASM/); (ii), 184 mouse fecal samples [32]; and (iii), 290 pig fecal samples (287 in ref. [33], and 3 samples collected from a swine farm in China). All of these samples were performed DNA preparation, library preparation and wholemetagenome shotgun sequencing using the metagenomic-specific protocols, as detailedly described at [30-33], and all of the raw sequencing data were available in the GigaScience Database (http://www.gigadb.org/). Metagenomic samples were basically processed (e.g. high-quality reads extraction and host DNA contamination removing) using the MOCAT pipeline [61] under the bioinformatic platforms at Beijing Genomics Institute (BGI)-Shenzhen.

Metagenomic reads were assembled using MEGAHIT (a de novo assembler for large and complex metagenomic sequences) [62]. Based on highly conservative of A. muciniphila genomes, we therefore extracted putative A. muciniphila fragments from the assembled contigs of each metagenomic sample via aligned to the 40 known A. muciniphila genomes, using BLASTN search (identity $>85 \%$, align length $\geq 300 \mathrm{bp}$, and covering $>70 \%$ of the contig length; -qcov_hsp_perc parameter, 0.7). Notably, the putative $A$. muciniphila sequences from one sample might be derived from two or more A. muciniphila strains. To avoid this, for each sample, the read depths for all putative A. muciniphila fragments were calculated, and only samples with unimodal read depth distribution of these fragments (indicating that only one major A. muciniphila strain exists in that sample) were retained. Low depth $(<15 \mathrm{X})$ and inconsistent $(>3 \sigma)$ 
fragments on each remaining sample were further removed. Lastly, we obtained 106 unimodal putative $A$. muciniphila genomes with minimum read depth of $15 \mathrm{X}$ from 106 metagenomic samples. The completeness of the draft $A$. muciniphila genomes was estimated by comparing to the known $A$. muciniphila core-genome (see Results), and all of these genomes obtained satisfactory assembling completeness ( $>95 \%)$.

The A. muciniphila reads were extracted from 106 metagenomic samples via reads mapping to the corresponding A. muciniphila MAG (using BWA, identity $>95 \%$ ), and were used for SNP calling based on the before-mentioned method. The accurate quantify of $A$. muciniphila in human and other mammalian gut microbiomes was preformed via reads mapping to the available genomes (all 146 strains) using BWA (identity >95\%).

\section{Identification of lateral gene transfer}

For each isolates, the LGT gene was identified via comparing its genes to all external Akkermansia genomes from the NCBI database using BLASTN (identity threshold of $85 \%$, covering $>90 \%$ of the gene length; -qcov_hsp_perc parameter, 0.9). Genomic fragment, which contains multiple nearby LGT genes derived from the same taxa, were further validated as a LGT event (representing a mobile genetic element or recombination event) via blasting to the origin genomic fragment of the origin taxa (overall similarity $>85 \%$ ).

\section{Bioinformatic analysis}

Functional profile of each A. muciniphila strain was generated using the number of genes that were annotated into the same function category (i.e. the KEGG pathway or CAZymes). And Principal component analysis (PCA) was performed on the functional profiles of all $\mathrm{A}$. muciniphila strains and visualized using the "ade4" package based on the $\mathrm{R}$ platform. For the comparative analysis, the stratification of age, gender, and BMI was adjusted based on the Generalized Linear Model (GLM, implemented in the R platform). Age and BMI were converted to categorical variables when performing the adjustment.

\section{Additional files}

Additional file 1: Table S1. Isolation, sequencing, assembly and gene prediction information of 39 A. muciniphila isolates and strain ATCC BAA835. (XLSX $16 \mathrm{~kb})$

Additional file 2: Figure S1. Pangenome "openness" of A. muciniphila. Figure S2. Principal components analysis shows the clustering of $A$. muciniphila isolates. Figure S3. Functional composition of the A. muciniphila pangenome. Figure S4. Principal components analysis on CAZymes profiles of $A$. muciniphila isolates. Figure S5. Relative abundance of A. muciniphila phylogroups in 368 Chinese gut microbiome samples. Figure S6. Gene flow and recombination events in A. muciniphila phylogroups. Figure S7. Loss and gain of orthologous groups during A. muciniphila evolution. (DOCX $682 \mathrm{~kb}$ )
Additional file 3: Table S2. Genome information of the 106 constructed A. muciniphila MAGs which are assembled from human and other mammalian gut microbiotas. (XLSX $17 \mathrm{~kb}$ )

Additional file 4: Table S3. Candidate horizontal gene transfer events identified from A. muciniphila genomes. (XLSX $11 \mathrm{~kb}$ )

\section{Abbreviations}

ANI: average nucleotide identity; BMl: body mass index;

CAZymes: carbohydrate-active enzymes; eggNOG: evolutionary genealogy of genes: non-supervised orthologous groups; KEGG: Kyoto encyclopedia of genes and genomes; LGT: lateral gene transfer; MAG: metagenome assembled genome; PCA: principal components analysis; SNP: single nucleotide polymorphism

\section{Acknowledgements}

Not applicable.

\section{Funding}

This work was supported by grants from the National Natural Science Foundation of China (No. 81441066), the Natural Science Foundation of Guangdong Province (S2013010014850), the Guangdong Provincial Department of Science and Technology (2015A020213002), and Guangzhou Kangze Medical Science and Technology Co., Ltd. Fund (201511). The funding bodies played no role in the design of the study and collection, analysis and interpretation of the data, or in writing of the manuscript.

\section{Availability of data and materials}

The raw sequencing data and assembled genome sequences reported in this article have been deposited in the NCBI BioProject PRJNA331216. The phylogenetic data of $A$. muciniphila strains reported in this article are available at the TreeBASE database with accession number TB2:S21535. The metagenomic datasets used in the current study are available from the corresponding author on reasonable request.

\section{Authors' contributions}

P.Y., G.X. and L.S. conducted the study. G.X., Z.J., W.F., W.D., Z.M. and O.Z. performed the experiments. G.X., L.S., and Y.Q. performed the DNA extraction and Illumina sequencing. G.X., L.S., L.X., J.Z. and L.P. performed the bioinformatic analyses. P.Y., L.S., L.X., and Y.J., wrote and edited the manuscript. All authors read and approved the final manuscript.

\section{Ethics approval and consent to participate}

This study received approval from the Medical Ethics Committee of Zhujiang Hospital of Southern Medical University (2014-JYYXB-009, for the use of human material) and the Southern Medical University Experimental Animal Ethics Committee (2015-057, for the use of animal material). Written informed consent for the scientific use of biological sample and relevant information was obtained from all human participants. The mice were derived from the Guangdong Medical Laboratory Animal Center (Guangzhou, China). The animal experiments were carried out in accordance with the approved guidelines of the ethics committee.

Consent for publication

Not applicable.

Competing interests

The authors declare that they have no competing interests.

\section{Publisher's Note}

Springer Nature remains neutral with regard to jurisdictional claims in published maps and institutional affiliations.

\section{Author details}

'Department of Laboratory Medicine, Zhujiang Hospital, Southern Medical University, Guangzhou 510282, China. 'Shenzhen Puensum Genetech Institute, Shenzhen 518052, China. ${ }^{3}$ Department of Biochemistry and Molecular Biology, Dalian Medical University, Dalian 116044, China. ${ }^{4}$ Guangzhou Kangze Medical Science and Technology Co., Ltd, Guangzhou 510630, China. 
Received: 1 March 2017 Accepted: 8 October 2017

Published online: 18 October 2017

\section{References}

1. Derrien M, Collado MC, Ben-amor K, Salminen S, de Vos WM. the Mucin degrader Akkermansia muciniphila is an abundant resident of the human intestinal tract. Appl Environ Microbiol. 2008;74(5):1646-8.

2. Le Chatelier E, Nielsen T, Qin J, Prifti E, Hildebrand F, Falony G, Almeida M, Arumugam M, Batto JM, Kennedy S, et al. Richness of human gut microbiome correlates with metabolic markers. Nature. 2013;500(7464):541-6.

3. Falony G, Joossens M, Vieira-Silva S, Wang J, Darzi Y, Faust K, Kurilshikov A Bonder MJ, Valles-Colomer M, Vandeputte D, et al. Population-level analysis of gut microbiome variation. Science. 2016;352(6285):560-4.

4. Backhed F, Roswall J, Peng Y, Feng Q, Jia H, Kovatcheva-Datchary P, Li Y, Xia Y, Xie $\mathrm{H}$, Zhong $\mathrm{H}$, et al. Dynamics and stabilization of the human gut microbiome during the first year of life. Cell Host Microbe. 2015;17(6):852

5. Collado MC, Derrien M, Isolauri E, de Vos WM, Salminen S. Intestinal integrity and Akkermansia muciniphila, a mucin-degrading member of the intestinal microbiota present in infants, adults, and the elderly. Appl Environ Microbiol. 2007:73(23):7767-70

6. Ley RE, Hamady M, Lozupone C, Turnbaugh PJ, Ramey RR, Bircher JS, Schlegel ML, Tucker TA, Schrenzel MD, Knight R, et al. Evolution of mammals and their gut microbes. Science. 2008;320(5883):1647-51.

7. Presley LL, Wei B, Braun J, Borneman J. Bacteria associated with immunoregulatory cells in mice. Appl Environ Microbiol. 2010;76(3):936-41.

8. Belzer C, de Vos WM. Microbes inside-from diversity to function: the case of Akkermansia. The ISME journal. 2012;6(8):1449-58.

9. Roeselers G, Mittge EK, Stephens WZ, Parichy DM, Cavanaugh CM, Guillemin K, Rawls JF. Evidence for a core gut microbiota in the zebrafish. The ISME journal. 2011;5(10):1595-608.

10. Derrien M, Vaughan EE, Plugge CM, de Vos WM. Akkermansia muciniphila gen. Nov., sp. nov., a human intestinal mucin-degrading bacterium. Int J Syst Evol Microbiol. 2004;54(Pt 5):1469-76.

11. Reunanen J, Kainulainen V, Huuskonen L, Ottman N, Belzer C, Huhtinen H, de Vos WM, Satokari R. Akkermansia muciniphila adheres to enterocytes and strengthens the integrity of the epithelial cell layer. Appl Environ Microbiol. 2015:81(11):3655-62.

12. Derrien M, Van Baarlen P, Hooiveld G, Norin E, Muller M, de Vos WM. Modulation of mucosal immune response, tolerance, and proliferation in mice colonized by the Mucin-degrader Akkermansia muciniphila. Front Microbiol. 2011;2:166

13. Derrien M, Belzer C, de Vos WM. Akkermansia muciniphila and its role in regulating host functions. Microb Pathog. 2016;

14. Everard A, Belzer C, Geurts L, Ouwerkerk JP, Druart C, Bindels LB, Guiot Y, Derrien M, Muccioli GG, Delzenne NM, et al. Cross-talk between Akkermansia muciniphila and intestinal epithelium controls diet-induced obesity. Proc Natl Acad Sci U S A. 2013;110(22):9066-71.

15. Schneeberger M, Everard A, Gomez-Valades AG, Matamoros S, Ramirez S Delzenne NM, Gomis R, Claret M, Cani PD. Akkermansia muciniphila inversely correlates with the onset of inflammation, altered adipose tissue metabolism and metabolic disorders during obesity in mice. Sci Rep. 2015;5:16643.

16. Shin NR, Lee JC, Lee HY, Kim MS, Whon TW, Lee MS, Bae JW. An increase in the Akkermansia spp. population induced by metformin treatment improves glucose homeostasis in diet-induced obese mice. Gut. 2014;63(5):727-35.

17. Karlsson CL, Onnerfalt J, Xu J, Molin G, Ahrne S, Thorngren-Jerneck K. The microbiota of the gut in preschool children with normal and excessive body weight. Obesity. 2012;20(11):2257-61.

18. Dao MC, Everard A, Aron-Wisnewsky J, Sokolovska N, Prifti E, Verger EO, Kayser BD, Levenez F, Chilloux J, Hoyles L, et al. Akkermansia muciniphila and improved metabolic health during a dietary intervention in obesity: relationship with gut microbiome richness and ecology. Gut. 2016;65(3):426-36.

19. Hansen $\mathrm{CH}$, Krych L, Nielsen DS, Vogensen FK, Hansen LH, Sorensen SJ, Buschard K, Hansen AK. Early life treatment with vancomycin propagates Akkermansia muciniphila and reduces diabetes incidence in the NOD mouse. Diabetologia. 2012;55(8):2285-94.

20. Png CW, Linden SK, Gilshenan KS, Zoetendal EG, McSweeney CS, Sly LI, McGuckin MA, Florin TH. Mucolytic bacteria with increased prevalence in IBD mucosa augment in vitro utilization of mucin by other bacteria. Am J Gastroenterol. 2010;105(11):2420-8.

21. Wang L, Christophersen CT, Sorich MJ, Gerber JP, Angley MT, Conlon MA. Low relative abundances of the mucolytic bacterium Akkermansia muciniphila and Bifidobacterium spp. in feces of children with autism. Appl Environ Microbiol. 2011:77(18):6718-21.

22. Qin J, Li Y, Cai Z, Li S, Zhu J, Zhang F, Liang S, Zhang W, Guan Y, Shen D, et al. A metagenome-wide association study of gut microbiota in type 2 diabetes. Nature. 2012;490(7418):55-60.

23. Weir TL, Manter DK, Sheflin AM, Barnett BA, Heuberger AL, Ryan EP. Stool microbiome and metabolome differences between colorectal cancer patients and healthy adults. PLoS One. 2013;8(8):e70803.

24. van Passel MW, Kant R, Zoetendal EG, Plugge CM, Derrien M, Malfatti SA, Chain PS, Woyke T, Palva A, de Vos WM, et al. The genome of Akkermansia muciniphila, a dedicated intestinal mucin degrader, and its use in exploring intestinal metagenomes. PLoS One. 2011;6(3):e16876.

25. Guo X, Zhang J, Wu F, Zhang M, Yi M, Peng Y. Different subtype strains of Akkermansia muciniphila abundantly colonize in southern China. J Appl Microbiol. 2016:120(2):452-9.

26. Schloissnig S, Arumugam M, Sunagawa S, Mitreva M, Tap J, Zhu A, Waller A Mende DR, Kultima JR, Martin J, et al. Genomic variation landscape of the human gut microbiome. Nature. 2013;493(7430):45-50.

27. Richter M, Rossello-Mora R. Shifting the genomic gold standard for the prokaryotic species definition. Proc Natl Acad Sci U S A. 2009;106(45):19126-31.

28. Huerta-Cepas J, Szklarczyk D, Forslund K, Cook H, Heller D, Walter MC, Rattei T, Mende DR, Sunagawa S, Kuhn M, et al. eggNOG 4.5: a hierarchical orthology framework with improved functional annotations for eukaryotic, prokaryotic and viral sequences. Nucleic Acids Res. 2016:44(D1):D286-93.

29. Kanehisa M, Goto S, Sato Y, Kawashima M, Furumichi M, Tanabe M. Data, information, knowledge and principle: back to metabolism in KEGG. Nucleic Acids Res. 2014;42(Database issue):D199-205.

30. Li J, Jia H, Cai X, Zhong H, Feng Q, Sunagawa S, Arumugam M, Kultima JR, Prifti $E$, Nielsen $T$, et al. An integrated catalog of reference genes in the human gut microbiome. Nat Biotechnol. 2014;32(8):834-41.

31. Human Microbiome Project C. A framework for human microbiome research. Nature. 2012:486(7402):215-21.

32. Xiao L, Feng Q, Liang S, Sonne SB, Xia Z, Qiu X, Li X, Long H, Zhang J, Zhang $D$, et al. A catalog of the mouse gut metagenome. Nat Biotechnol. 2015;33(10):1103-8.

33. Xiao L, Estelle J, Kiilerich $P$, Ramayo-Caldas Y, Xia Z, Feng Q, Liang S, Pedersen $\mathrm{AO}$, Kjeldsen NJ, Liu C, et al. A reference gene catalogue of the pig gut microbiome. Nature microbiology. 2016:16161.

34. Li J, Lin S, Vanhoutte PM, Woo CW, Xu A. Akkermansia Muciniphila protects against atherosclerosis by preventing metabolic Endotoxemia-induced inflammation in Apoe-/- mice. Circulation. 2016;133(24):2434-46.

35. de la Cuesta-Zuluaga J, Mueller NT, Corrales-Agudelo V, Velasquez-Mejia EP, Carmona JA, Abad JM, Escobar JS. Metformin is associated with higher relative abundance of Mucin-degrading Akkermansia muciniphila and several short-chain fatty acid-producing microbiota in the gut. Diabetes Care. 2017;40(1):54-62.

36. Sangwan $\mathrm{P}$, Chen $\mathrm{X}$, Hugenholtz $\mathrm{P}$, Janssen PH. Chthoniobacter flavus gen. Nov., sp. nov., the first pure-culture representative of subdivision two, Spartobacteria classis nov., of the phylum Verrucomicrobia. Appl Environ Microbiol. 2004:70(10):5875-81.

37. Yee B, Lafi FF, Oakley B, Staley JT, Fuerst JA. A canonical FtsZ protein in Verrucomicrobium spinosum, a member of the bacterial phylum Verrucomicrobia that also includes tubulin-producing Prosthecobacter species. BMC Evol Biol. 2007:7:37.

38. Gomez-Gallego C, Pohl S, Salminen S, De Vos WM, Kneifel W. Akkermansia muciniphila: a novel functional microbe with probiotic properties. Benefic Microbes. 2016;7(4):571-84

39. Van Herreweghen F, Van den Abbeele P, De Mulder T, De Weirdt R, Geirnaert A, Hernandez-Sanabria E, Vilchez-Vargas R, Jauregui R, Pieper DH, Belzer C, et al. Vitro colonisation of the distal colon by Akkermansia muciniphila is largely mucin and pH dependent. Beneficial microbes; 2016. p. 1-16.

40. El Kaoutari A, Armougom F, Gordon Jl, Raoult D, Henrissat B. The abundance and variety of carbohydrate-active enzymes in the human gut microbiota. Nature reviews. Microbiology. 2013;11(7):497-504.

41. Scholz P, Haring V, Wittmann-Liebold B, Ashman K, Bagdasarian M, Scherzinger $\mathrm{E}$. Complete nucleotide sequence and gene organization of the broad-host-range plasmid RSF1010. Gene. 1989:75(2):271-88.

42. Meyer R. Replication and conjugative mobilization of broad host-range IncQ plasmids. Plasmid. 2009:62(2):57-70.

43. Daly M, Villa L, Pezzella C, Fanning S, Carattoli A. Comparison of multidrug resistance gene regions between two geographically unrelated salmonella serotypes. J Antimicrob Chemother. 2005;55(4):558-61. 
44. Ramirez MS, Tolmasky ME. Aminoglycoside modifying enzymes. Drug resistance updates : reviews and commentaries in antimicrobial and anticancer chemotherapy. 2010;13(6):151-71.

45. FASTX-Toolkit: FASTQ/A short-reads pre-processing tools [http://hannonlab. cshl.edu/fastx_toolkit].

46. Zerbino DR, Birney E. Velvet: algorithms for de novo short read assembly using de Bruijn graphs. Genome Res. 2008;18(5):821-9.

47. Boetzer M, Henkel CV, Jansen HJ, Butler D, Pirovano W. Scaffolding pre-assembled contigs using SSPACE. Bioinformatics. 2011;27(4):578-9.

48. Nadalin F, Vezzi F, Policriti A: GapFiller: a de novo assembly approach to fill the gap within paired reads. BMC bioinformatics 2012, 13 Suppl 14:S8.

49. Seemann T. Prokka: rapid prokaryotic genome annotation. Bioinformatics. 2014;30(14):2068-9.

50. Lagesen K, Hallin P, Rodland EA, Staerfeldt HH, Rognes T, Ussery DW. RNAmmer: consistent and rapid annotation of ribosomal RNA genes. Nucleic Acids Res. 2007;35(9):3100-8.

51. Laslett $D$, Canback B. ARAGORN, a program to detect tRNA genes and tmRNA genes in nucleotide sequences. Nucleic Acids Res. 2004;32(1):11-6.

52. Minced: Mining CRISPRs in Environmental Datasets [https://github.com/ ctSkennerton/minced/tree/master].

53. Hyatt $D$, Chen GL, Locascio PF, Land ML, Larimer FW, Hauser L. Prodigal: prokaryotic gene recognition and translation initiation site identification. BMC bioinformatics. 2010;11:119.

54. Cantarel BL, Coutinho PM, Rancurel C, Bernard T, Lombard V, Henrissat B. The carbohydrate-active EnZymes database (CAZy): an expert resource for Glycogenomics. Nucleic Acids Res. 2009;37(Database issue):D233-8.

55. Page AJ, Cummins CA, Hunt M, Wong VK, Reuter S, Holden MT, Fookes M, Falush D, Keane JA, Parkhill J. Roary: rapid large-scale prokaryote pan genome analysis. Bioinformatics. 2015;31(22):3691-3.

56. Goris J, Konstantinidis KT, Klappenbach JA, Coenye T, Vandamme P, Tiedje JM. DNA-DNA hybridization values and their relationship to whole-genome sequence similarities. Int J Syst Evol Microbiol. 2007;57(Pt 1):81-91.

57. Li H, Durbin R. Fast and accurate short read alignment with burrowswheeler transform. Bioinformatics. 2009;25(14):1754-60

58. Li H, Handsaker B, Wysoker A, Fennell T, Ruan J, Homer N, Marth G, Abecasis G, Durbin R. Genome project data processing S: the sequence alignment/ map format and SAMtools. Bioinformatics. 2009:25(16):2078-9.

59. Stamatakis A. RAxML version 8: a tool for phylogenetic analysis and postanalysis of large phylogenies. Bioinformatics. 2014;30(9):1312-3.

60. Kumar S, Stecher G, Tamura K. MEGA7: molecular evolutionary genetics analysis version 7.0 for bigger datasets. Mol Biol Evol. 2016;33(7):1870-4.

61. Kultima JR, Sunagawa S, Li J, Chen W, Chen H, Mende DR, Arumugam M, Pan Q, Liu B, Qin J, et al. MOCAT: a metagenomics assembly and gene prediction toolkit. PLoS One. 2012;7(10):e47656.

62. Li D, Liu CM, Luo R, Sadakane K, Lam TW. MEGAHIT: an ultra-fast singlenode solution for large and complex metagenomics assembly via succinct de Bruijn graph. Bioinformatics. 2015;31(10):1674-6.

\section{Submit your next manuscript to BioMed Central and we will help you at every step:}

- We accept pre-submission inquiries

- Our selector tool helps you to find the most relevant journal

- We provide round the clock customer support

- Convenient online submission

- Thorough peer review

- Inclusion in PubMed and all major indexing services

- Maximum visibility for your research

Submit your manuscript at www.biomedcentral.com/submit

C) Biomed Central 